\title{
Failure-Oriented-Accelerated-Testing and its Possible Application in Ergonomics
}

\section{Suhir $\mathrm{E}^{*}$}

Department of Applied Electronic Materials, Technical University, Austria

*Corresponding author: E. Suhir, Department of Applied Electronic Materials, Institution of Sensors and Actuators, Technical University, Vienna, Austria; and ERS Co., 727 Alvina Ct., Los Altos, CA 94024, Austria, Tel: 408-410-0886; Email: suhire@aol.com

\section{Commentary}

Failure-oriented-accelerated-testing (FOAT) [1] has been suggested as part of the probabilistic-design-forreliability (PDfR) concept several years ago in application to microelectronics reliability assurance [2]. It has been argued that when reliability of a material or a device is critical, it has to be quantified to be assured, and, because nothing is perfect, such a quantification should be done on the probabilistic basis [3]: the difference between a highly reliable product or a system and an insufficiently reliably one is "merely" in the level of their never-zero probability of failure. The concept of the probabilistic predictive modeling has been recently applied to various "human-inthe-loop" problems in engineering: the need of assuring adequate performance of both equipment/instrumentation and humans is particularly imperative in this area of human activity [4]. It has been recently suggested that flight simulator can be employed as an appropriate test vehicle that could be used to quantify, on the probabilistic basis, the required level of the human capacity factor (HCF) with respect to the expected mental workload (MWL) during fulfillment of a particular aerospace mission or when encountering an extraordinary/off-normal situation [5]. The general point is that in any significant ergonomics effort an appropriate accelerated test vehicle (ATV) and FOAT should be used if there is intent to predict the outcome of an important ergonomics effort. Let us show, as an example, how this could be done.
In accordance with one of the modifications of the doubleexponential-probability distribution function (DEPDF) [6], the probability of non-failure of a human, when fulfilling a particular mission/task, can be sought in the form:

$$
P=\exp \left[-\gamma M_{*} \exp \left(-\frac{F}{G}\right)\right]
$$

Here $F$ is the HCF, a long-term characteristic of the performer under test (a detailed description of which human qualities should/could be included into this factor could be found in [7]), $G$ is the MWL ([8]), $t$ is time, $M$ is the measured (monitored) parameter of human performance during the accelerated test, it's between $M_{*}$ value is an indication of a failure/error, and $\gamma$ is the sensitivity factor that should be evaluated during the adequately designed, organized and conducted FOAT on an appropriate test vehicle (such as, say, flight simulator). It is assumed that the formula (1) is valid for both the accelerated testing and in the actual operating conditions.

From (1) we obtain:

$$
\frac{d P}{d t}=-\frac{H(P)}{t},(2)
$$

where $H(P)=P \ln P$ is the entropy of the distribution (1). Thus, the function (1) reflects the underlying rationale behind this distribution: the distribution (1) reflects an assumption that the change of the probability of non-failure with time is proportional to the entropy of this distribution and is inversely proportional to time. 


\section{Ergonomics International Journal}

When conducting a FOAT aimed at the evaluation of the sensitivity parameter $\gamma$, one should bring together a group of more or less equally (preferably highly) qualified individuals, and proceed from the fact that the HCF is a long-term characteristic that remains unchanged for these individuals during the relatively short FOAT time. The MWL, on the other hand, is a short-term characteristic that can be tailored, in many ways, depending on the anticipated MWL conditions during accelerated testing.

Solving the equation (1) for the HCF $F$, the following condition can be obtained:

$$
-G \ln \left(\frac{n}{\gamma}\right)=F=\text { Const. , (3) }
$$

where

$$
n=-\frac{\ln P}{t M_{*}} .
$$

Let the FOAT is conducted at two MWL levels, $G_{1}$ and $G_{2}$, and the criterion $M_{*}$ was observed and recorded at the times of $t_{1}$ and $t_{2}$ for the established percentages of $P_{1}$ and $P_{2}$ of individuals who did not fail during the test. Then the condition (3) enables to obtain the following formula for the $\gamma$ value:

$$
\begin{aligned}
& \gamma=\exp \left(\frac{\ln n_{2}-\frac{G_{1}}{G_{2}} \ln n_{1}}{1-\frac{G_{1}}{G_{2}}}\right)(5) \quad n_{2}=-\frac{\ln P_{2}}{t_{2} M_{*}}=-\frac{\ln 0.5}{1.5 \times 120}=38.5082 \times 1 \\
& \gamma=\exp \left(\frac{\ln n_{2}-\frac{G_{1}}{G_{2}} \ln n_{1}}{1-\frac{G_{1}}{G_{2}}}\right)=\exp \left(\frac{\ln 38.5082 \times 10^{-4}-0.5 \ln 9.2976 \times 10^{-4}}{1-0.5}\right)=0.015948
\end{aligned}
$$

Then the equation (1) can be written as follows:

$$
P=\exp \left[-0.015948 \times 120 t \exp \left(-\frac{F}{G}\right)\right]=\exp \left[-1.9138 t \exp \left(-\frac{F}{G}\right)\right]
$$

Suhir E, et al. Failure-Oriented-Accelerated-Testing and its Possible Application in Ergonomics. Ergonomics Int J 2019, 3(2): 000199.
HCF of the individuals that underwent the accelerated testing can be determined, in accordance with the formula (3), as

$$
F=-G_{1} \ln \left(\frac{n_{1}}{\gamma}\right)=-G_{2} \ln \left(\frac{n_{2}}{\gamma}\right)
$$

Note that the HCF $F$ value is analogous in a way to the activation energy in the Arrhenius equation in the kinetic theory of chemical reactions, and the MWL G is analogous to the thermal energy, calculated as a product of Boltzmann constant and the absolute temperature.

Let, e.g., the same group of individuals was tested at two different MWL levels, $G_{1}$ and $G_{2}$, until failure (whatever its definition and nature might be), and let the load ratio was $\frac{G_{2}}{G_{1}}=2$. Because of that the time to failure (TTF) was considerably shorter and the number of the failed individuals was considerably larger, for the same $M_{*}$ level (say, $M_{*}=120$ ), in the second round of tests.

Let, e.g., $P_{1}=0.8, P_{2}=0.5, t_{1}=2.0 h$, and $t_{2}=1.5 h$. Then

$$
\begin{gathered}
n_{1}=-\frac{\ln P_{1}}{t_{1} M_{*}}=-\frac{\ln 0.8}{2 \times 120}=9.2976 \times 10^{-4}, \\
n_{2}=-\frac{\ln P_{2}}{t_{2} M_{*}}=-\frac{\ln 0.5}{1.5 \times 120}=38.5082 \times 10^{-4},
\end{gathered}
$$


The calculated probabilities of non-failure for a mission lasting for, say, $48 \mathrm{~h}$ are as follows (Table 1):

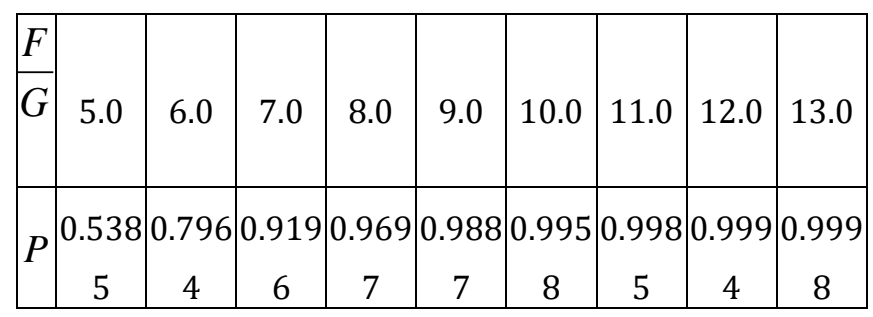

Table 1: Probabilities of non-failure.

Thus, in this example, the HCF should exceed considerably the MWL to make the probability of the human nonfailure high enough.

\section{References}

1. Suhir E (2013) Failure-Oriented-Accelerated-Testing (FOAT) and Its Role in Making a Viable IC Package into a Reliable Product, Circuits Assembly.
2. Suhir E (2010) Probabilistic Design for Reliabilit. Chip Scale Reviews 4(6).

3. Suhir E (2014) Electronics Reliability Cannot Be Assured, if it is not quantified. Chip Scale Reviews.

4. Suhir E (2018) Human-in-the-Loop: Probabilistic Modeling of an Aerospace Mission Outcome. CRC Press.

5. Suhir E (2019) Assessment of the Required Human Capacity Factor (HCF) Using Flight Simulator as an Appropriate Accelerated Test Vehicle. Int J of Human Factor Modeling and Simulation.

6. Suhir E (2017) Human-in-the-Loop: Application of the Double Exponential Probability Distribution Function Enables to Quantify the Role of the Human Factor. Int $J$ of Human Factor Modeling and Simulation 5(4).

7. Suhir E (2013) Miracle-on-the-Hudson: Quantified Aftermath. Int $\mathrm{J}$ of Human Factors Modeling and Simulation 4(1).

8. Charles RL, Nixon J (2019) Measuring Mental Workload Using Physiological Measures: A Systematic Review. Applied Ergonomics 74: 221-232. 